\title{
Cervical dermoid cyst
}

INSERM

\section{Source}

INSERM. (1999). Orphanet: an online rare disease and orphan drug data base. Cervical dermoid cyst. ORPHA:141046

Cervical dermoid cyst is a rare, benign cutaneous neoplasm containing keratinized epithelium and dermal derivatives, such as hair follicles, sweat and sebaceous glands, smooth muscle or fibroadipose tissue which usually manifests as a slow-growing, painless mass in the submandibular or sublingual space. Depending on the location, and especially after sudden enlargement, it can cause dyspnea, dysphagia or dysphonia. 